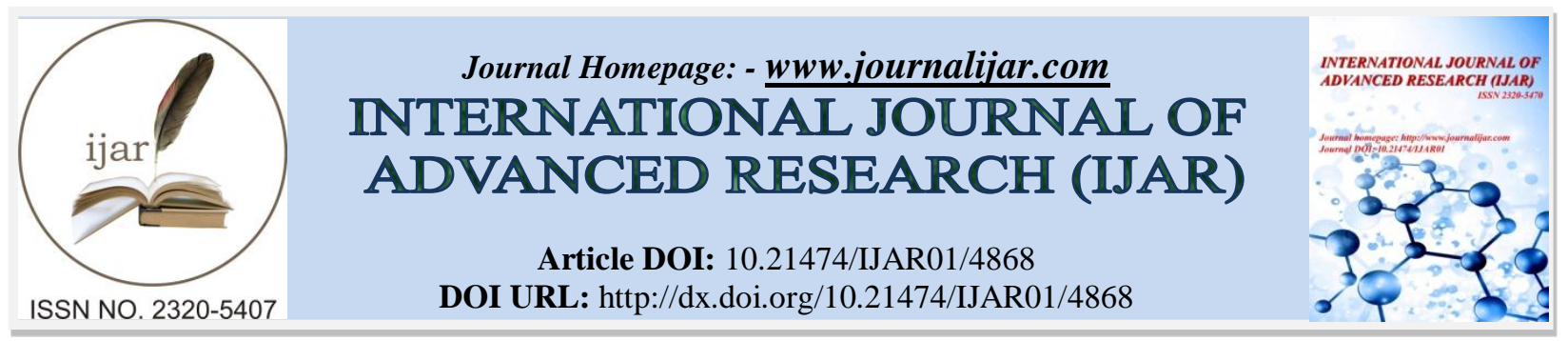

RESEARCH ARTICLE

\title{
INVESTIGATIONS ON THE EFFECT OF AMINO ACID DOPING ON THE GROWTH AND CHARACTERIZATION OF ALKALI METAL ACID PHTHALATE SINGLE CRYSTALS.
}

\author{
S. Shek Dhavud ${ }^{1}$ and ${ }^{*} J$. Thomas Joseph Prakash ${ }^{2}$. \\ 1. PG and Research Department of Physics, Jamal Mohamed College (Autonomous), Affiliated to Bharathidasan \\ University, Trichy-20, Tamilnadu, India. \\ 2. PG and Research Department of Physics, Government Arts College, Affiliated to Bharathidasan University, \\ Trichy-22, Tamilnadu, India.
}

\section{Manuscript Info}

Manuscript History

Received: 17 May 2017

Final Accepted: 19 June 2017

Published: July 2017

Key words:-

Synthesis,

Crystal structure,

Photoluminescence,

Antibacterial activity,

\section{Abstract}

Undoped and $0.5 \mathrm{~mol} \%$ of L-aspartic acid $\left(\mathrm{LA}-\mathrm{C}_{4} \mathrm{H}_{7} \mathrm{NO}_{4}\right)$ and L-threonine $\left(\mathrm{LT}-\mathrm{C}_{4} \mathrm{H}_{9} \mathrm{NO}_{3}\right)$ doped sodium acid phthalate (NaAP) single crystals were successfully grown from aqueous solution by slow evaporation solution growth technique. Single X-ray diffraction revealed that the grown crystals possess orthorhombic crystal structure and revealed lattice distortion due to impurities. The crystallinity nature of the grown crystals was analyzed by powder X-ray diffraction analysis. Functional groups and the modes of vibrations were identified by FT-IR spectral analysis. Optical transmittance shows that L-threonine doped NaAP crystal possesses higher transparency compared to undoped NaAP and L- aspartic acid doped NaAP and the band gap energy values of the grown crystals were calculated. The photoluminescence properties of the grown crystals were investigated. The mechanical strength of the grown crystals was estimated by microhardness studies. The second harmonic generation of the grown crystals was confirmed by the Kurtz and Perry technique. Antibacterial activity of the title compounds against Streptococcus pneumoniae, Escherichia coli, and Klebsiella pneumonia strains was studied.

Copy Right, IJAR, 2017,. All rights reserved.

\section{Introduction:-}

Semiorganic crystals are a new category of materials recently examined for their fascinating properties and stable physiochemical properties, which are significant for device fabrication and applied research [1,2]. The high nonlinearity, high resistance to laser-induced damage, low angular sensitivity and better mechanical hardness of semiorganic crystals incorporate in the strong NLO properties and chemical flexibility of organic materials with the physical sturdiness and excellent transmittance of inorganic materials [3-6]. In addition, the approach of high NLO efficiency organic molecules with the favorable physical properties of the inorganic materials has been an active area of research in the last two decades.

Organic material with high nonlinear optical coefficients combines with an inorganic material exhibiting excellent physical properties, giving semiorganic material. Hydrogen bonding of acid based interaction between organic cation and an inorganic anion of semiorganic give mechanical strong and thermally stable NLO crystals [7]. Highly 
polarizable acid-base interactions of organic and inorganic molecules are liable for NLO properties, as linking through a hydrogen bond network affords non-centrosymmetric structural systems [8,9]. Due to its wonderful physiochemical properties, the amino acid family of crystals has been subjected to extensive investigation by several research scientists. Particularly in the view of NLO applications, organic amino acid crystals are interesting because they contain a paired donor carboxylic $(\mathrm{COOH})$ group and proton acceptor $\left(\mathrm{NH}_{2}\right)$ group known as a zwitterion, which creates hydrogen bonds. This form of dipolar nature of amino acids demonstrates to be a perfect candidate for NLO applications. Amino acid dopants are reportable to increase the NLO and microhardness characteristics of materials. With this motivation, we've got chosen the amino acids, L-threonine, L-aspartic acid as dopants for NaAP single crystal.

In the current work, undoped NaAP, L-aspartic acid and L-threonine doped sodium acid phthalate (NaAP) crystals were grown successfully from aqueous solution by slow evaporation growth technique. The grown crystals characterized by single XRD, powder XRD, FTIR, UV, PL, SHG, Microhardness studies and Antibacterial activity were carried out. As a result, L-threonine doped NaAP crystal has superior one compare to other grown crystals.

\section{Experimental Details:-}

Sodium Acid Phthalate (NaAP) is synthesized by dissolving stoichiometric ratio of sodium bicarbonate (Merck, AR grade, 99.8\%) and phthalic acid (Merck, AR grade, $99 \%$ ) in deionized water. The solution was thoroughly stirred for $3 \mathrm{hrs}$ continuously using a magnetic stirrer till to get the homogeneous solution and the saturated solution was filtered using Whatmann no.1 filter paper and it was kept in undisturbed conditions. The quality of the grown crystals was enhanced by recrystallization process. After a duration of 35 days, transparent good quality of the crystal of undoped NaAP was harvested (Fig. 1a).

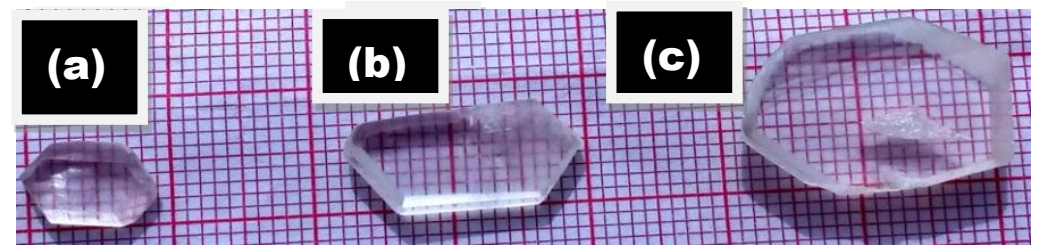

Figure 1a:- As grown crystal of the undoped NaAP

Figure 1b:- As grown crystal of L-aspartic acid doped NaAP

Figure 1c:- As grown crystal of L-threonine doped NaAP

0.5 Mole \% of L-aspartic acid and L-threonine and (Loba, AR grade, 99\%) was added to the prepared solution of NaAP separately and the solution was thoroughly stirred endlessly for 4 hrs till to get the homogeneous solution. The saturated solution was filtered using Whatmann no.1 filter paper and it was kept in undisturbed conditions. After a period of 43 and 45 days, transparent good quality of the crystal of L-aspartic acid doped NaAP (LA-NaAP) and Lthreonine doped NaAP (LT-NaAP) were harvested (Fig. 1b \& 1c) respectively.

\section{Results and Discussion:-}

X-ray diffraction studies:-

The single crystal X-ray diffraction analysis of the grown crystals was recorded ENRAF NONIUS CAD 4 diffractometer with $\mathrm{MoK} \alpha$ radiation $(\lambda=0.71073 \AA)$ to identify the unit cell parameters. This analysis has revealed that the single crystals of undoped NaAP, LA-NaAP and LT-NaAP crystallize in orthorhombic system with the space group B2ab. The calculated lattice parameters of the grown crystals are given in Table 1. From Table 1, the values of the lattice parameters of the LA-NaAP and LT-NaAP crystals are increased as compared to the undoped NaAP. This is due to the incorporation of L-threonine and L-aspartic acid in the NaAP crystal lattice. The calculated lattice parameter and unit cell volume are given in Table 1 and which is in good agreement with the early literature [10]. 
Table 1:- Lattice parameters of undoped and amino acids doped NaAP crystals

\begin{tabular}{|c|c|c|c|c|}
\hline Lattice parameters & Reported Value NaAP [10] & Undoped NaAP & LA-NaAP & LT-NaAP \\
\hline $\mathrm{a}(\AA)$ & 6.75 & 6.75 & 6.88 & 6.87 \\
\hline $\mathrm{b}(\AA)$ & 9.31 & 9.33 & 9.37 & 9.39 \\
\hline $\mathrm{c}(\AA)$ & 26.60 & 26.65 & 26.69 & 26.70 \\
\hline $\mathrm{V}(\AA)^{3}$ & 1671.61 & 1678.35 & 1682.18 & 1688.50 \\
\hline$\alpha=\beta=\gamma$ & \multicolumn{4}{|l}{} \\
\hline
\end{tabular}

Powder X-ray diffraction pattern of all the grown crystals was recorded on X'pert PRO powder X-ray diffraction $(40 \mathrm{kV})$ using $\mathrm{Cu}-\mathrm{K} \alpha$ radiation of wavelength $\lambda=1.5406 \AA$. Powder XRD patterns of undoped NaAP, LA-NaAP and LT-NaAP crystals are shown in Fig. 2. The XRD pattern shows the peak shift for the crystals grown in the presence of amino acid sources in comparison to the undoped NaAP and these shifts in peak positions caused a small modification in lattice parameters in comparison to the undoped NaAP. All the peaks were indexed by JCPDS software (card no: 32-1895 for pure NaAP).

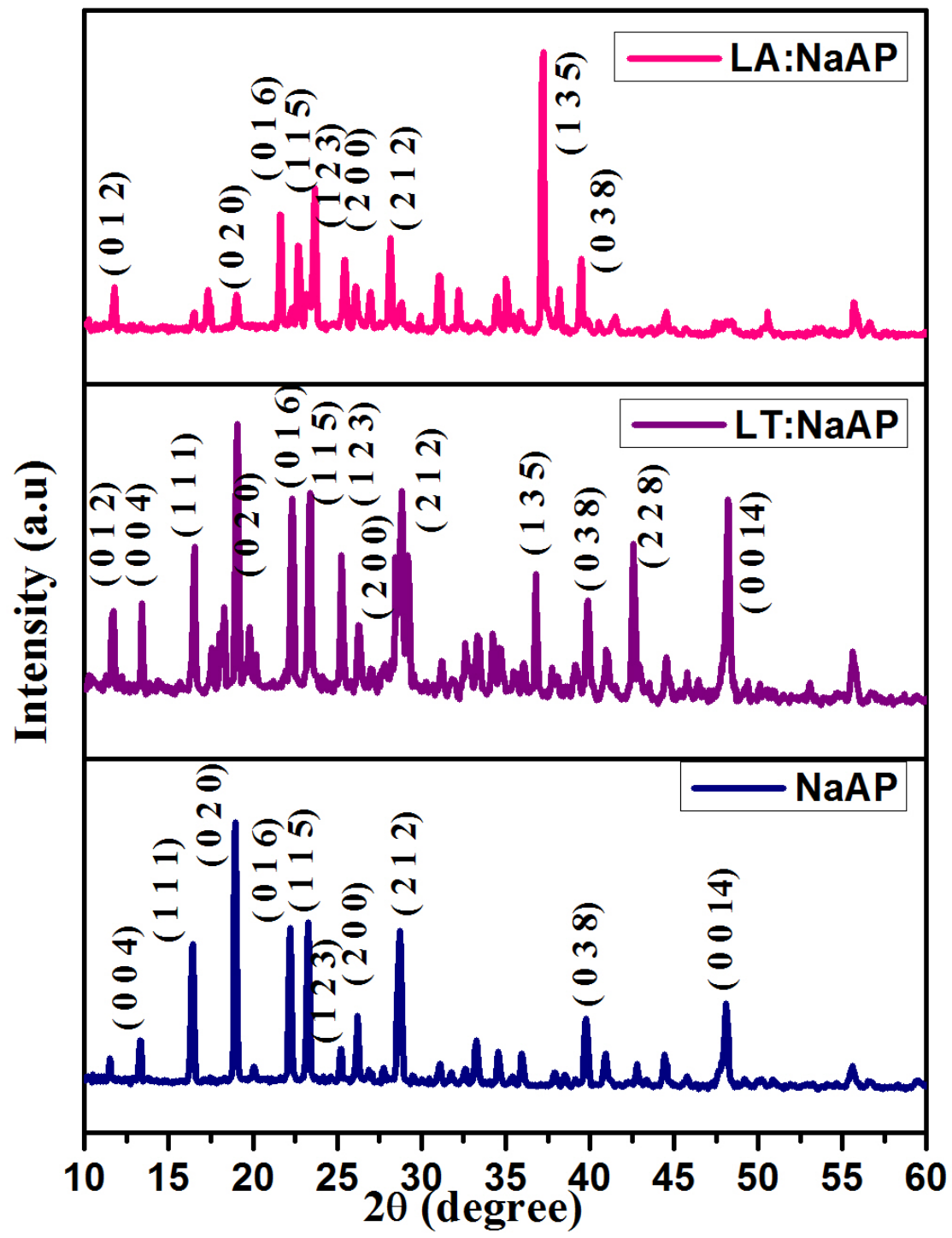

Figure 2:-. Powder XRD patterns of undoped NaAP, LT-NaAP and LA-NaAP crystals 
FT-IR spectral analysis:-

FT-IR transmittance spectra of the grown crystals were recorded in the range of 400-4000 $\mathrm{cm}^{-1}$ using Lamda 35 make Perkin Elmer (Spectrum RX1) spectrometer. The recorded FT-IR spectra and their vibrations are given in Fig. 3 and Table 2 respectively. The absorption peaks in the range of $3020-3650 \mathrm{~cm}^{-1}$ corresponds to the vibrational mode of O-H bonds. FTIR result shows that the strong O-H peak observed at $3505 \mathrm{~cm}^{-1}, 3492 \mathrm{~cm}^{-1}$ and $3496 \mathrm{~cm}^{-1}$ for undoped NaAP, LA-NaAP and LT-NaAP respectively. The phenyl ring C-C stretching band found at $1473 \mathrm{~cm}^{-1}$, $1477 \mathrm{~cm}^{-1}$ and $1475 \mathrm{~cm}^{-1}$ for undoped NaAP, LA-NaAP and LT-NaAP respectively. The C-H symmetric stretching bands attributed at $2459 \mathrm{~cm}^{-1}, 2471 \mathrm{~cm}^{-1}$ and $2478 \mathrm{~cm}^{-1}$ for undoped NaAP, LA-NaAP and LT-NaAP respectively. The $\mathrm{C}=\mathrm{O}$ stretching vibration are ascribed at $1603 \mathrm{~cm}^{-1}, 1606 \mathrm{~cm}^{-1}$ and $1609 \mathrm{~cm}^{-1}$ for undoped NaAP, LA-NaAP and LT-NaAP respectively. The group of C-H out-of-plane bending vibrations observed at $\left(861 \mathrm{~cm}^{-1}, 753 \mathrm{~cm}^{-1}, 645 \mathrm{~cm}^{-1}\right.$ \& $\left.590 \mathrm{~cm}^{-1}\right),\left(860 \mathrm{~cm}^{-1}, 754 \mathrm{~cm}^{-1}, 647 \mathrm{~cm}^{-1} \& 587 \mathrm{~cm}^{-1}\right)$ and $\left(866 \mathrm{~cm}^{-1}, 759 \mathrm{~cm}^{-1}, 649 \mathrm{~cm}^{-1} \& 559 \mathrm{~cm}^{-1}\right)$ for undoped NaAP, LA-NaAP and LT-NaAP respectively $[10,11]$. Very slight variations in the position of vibrational bands of NaAP were observed due to amino acids dopants.

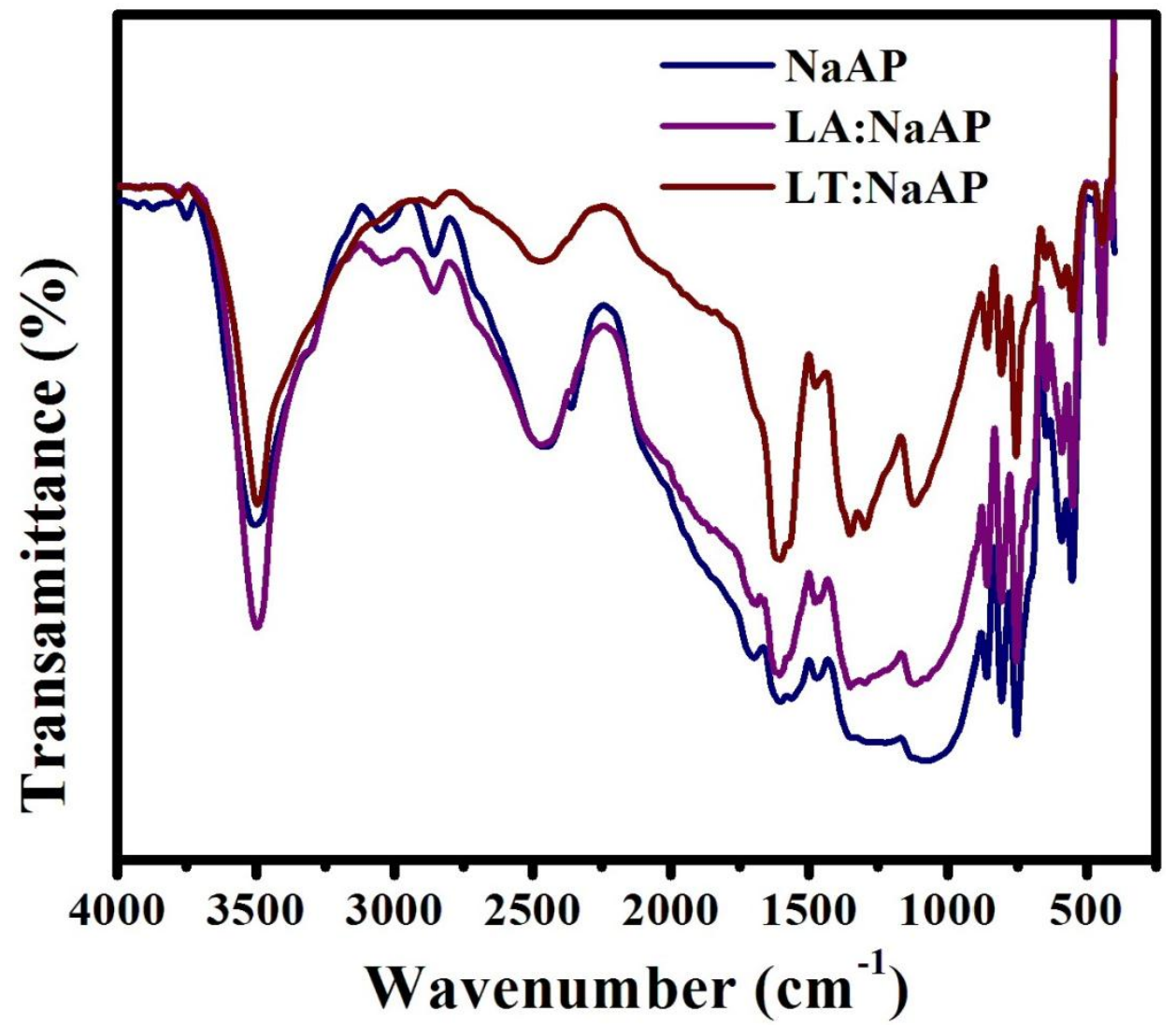

Figure 3:- FTIR spectra of undoped NaAP, LA-NaAP and LT-NaAP crystals

Table 2:- FTIR spectral assignments of undoped NaAP, LA-NaAP and LT-NaAP crystals

\begin{tabular}{|c|c|c|l|}
\hline \multicolumn{2}{|c|}{ Observed wavenumbers $\left(\mathbf{c m}^{-1}\right)$} & \multirow{2}{*}{ Assignments } \\
\hline Undoped NaAP & LA:NaAP & LT:NaAP & O-H stretching \\
\hline 3505 & 3492 & 3496 & C-H symmetric stretching \\
\hline 2459 & 2471 & 2478 & C=O stretching \\
\hline 1603 & 1606 & 1609 & C-C stretching \\
\hline 1473 & 1477 & 1475 & $\begin{array}{l}\text { C-H out of plane } \\
\text { deformation }\end{array}$ \\
\hline $861,753,645 \& 590$ & $860,754,647 \& 587$ & $866,759,649 \& 559$ & \\
\hline
\end{tabular}




\section{UV-Visible spectral analysis:-}

The UV-visible transmittance spectra of undoped NaAP, LA-NaAP and LT-NaAP crystals are shown in Fig. 4 recorded in the range of 190-1100 nm using PERKIN ELMER LAMBDA 35 UV-Visible spectrophotometer. For optical fabrication, the crystal should be highly transparent in the considerable region of wavelength [12,13]. The lower cut-off wavelength for the grown crystals was observed to be around $300 \mathrm{~nm}$. Undoped NaAP, LA-NaAP and LT-NaAP crystals possessed transmittance percentages $60 \%, 70 \%$ and $80 \%$ respectively. The good transmissions of the crystal in the entire visible region suggest its suitability for second harmonic generation devices [14,15]. The dependence of optical absorption coefficient on the photon energy helps to study the band structure and the type of transition of electrons [16].

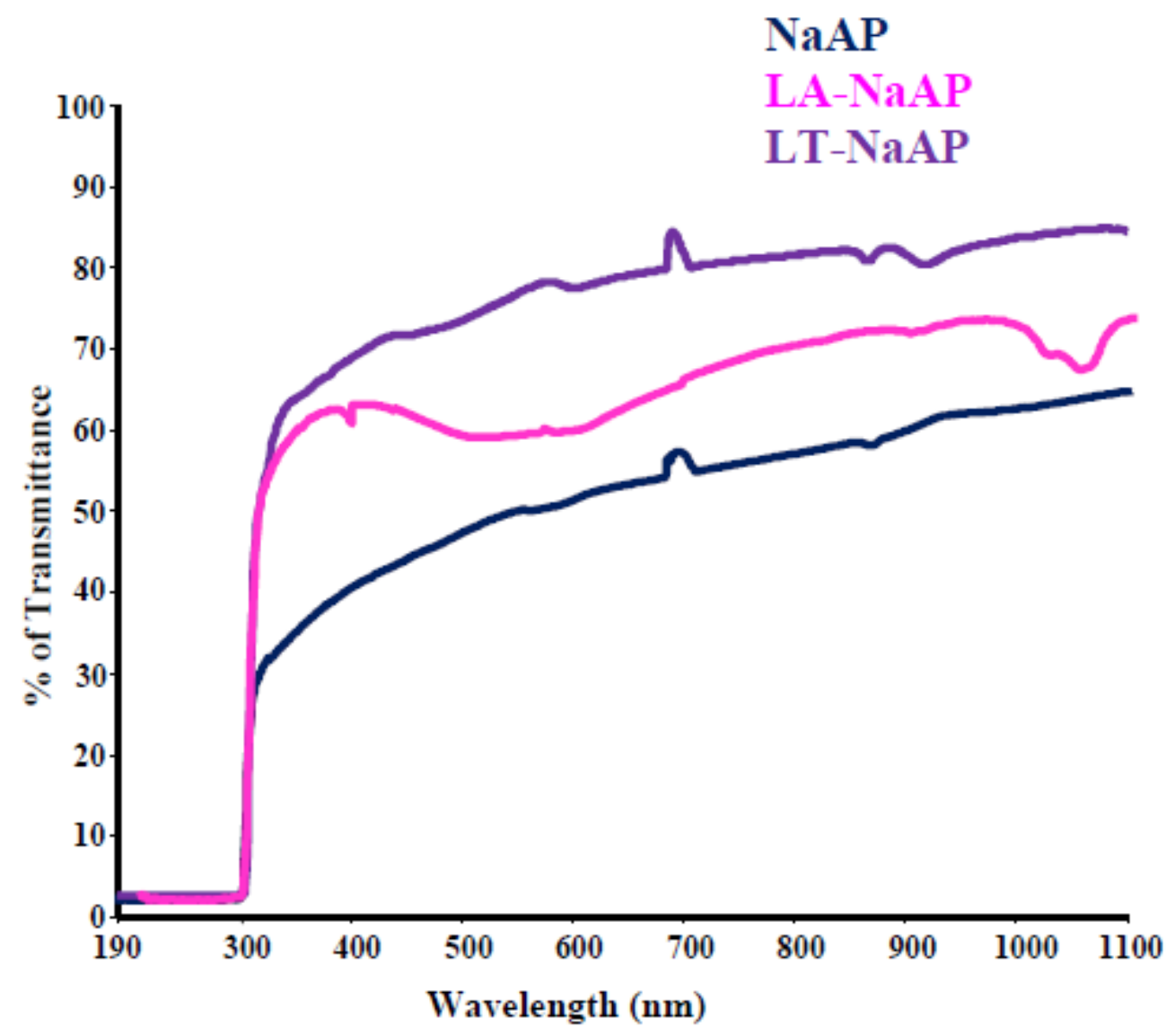

Figure 4:- UV-Visible transmission spectra of undoped, LA-NaAP and LT-NaAP crystals

The optical absorption coefficient $(\alpha)$ was calculated from the transmittance using the following relation

$$
\alpha=\frac{2.303 \log \left(\frac{1}{T}\right)}{d}
$$

Where, $\mathrm{T}$ - transmittance of the grown crystals, d - thickness of the grown crystals. As a direct band gap material, the crystal under study as an absorption coefficient $(\alpha)$ obeying the following equation for high photon energies $h v$

$$
\boldsymbol{\alpha} \boldsymbol{h} \boldsymbol{v}=\boldsymbol{A}(\boldsymbol{h} \boldsymbol{v}-\boldsymbol{E g})^{n}
$$

$------(2)$

Where A - energy independent constant, Eg - band gap corresponding to a particular transition and the exponent $\mathrm{n}$ characterizes the nature of the band transition. For crystalline semiconductors, $n=1 / 2,2,3 / 2,3$ values corresponding to the allowed direct, allowed indirect, forbidden direct and indirect transitions respectively.

Optical band gap value was calculated by the graph between $(\alpha h v)^{2}$ versus photon energy hv is shown in Fig. 5. The band gap obtained from the graph for undoped NaAP, LA-NaAP and LT-NaAP crystals are found to be $3.8 \mathrm{eV}$, $4.0 \mathrm{eV}$ and, $4.2 \mathrm{eV}$ respectively. As a consequence of wide band gap, LT-NaAP crystal has a large transmittance in the visible region [17]. 


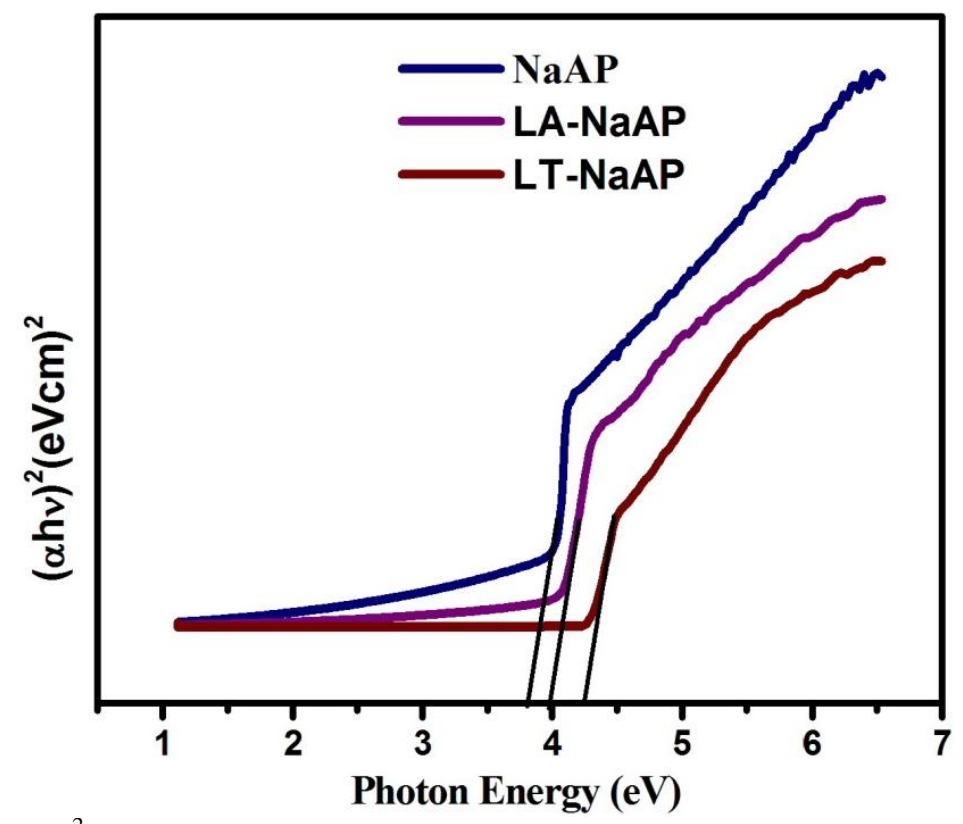

Figure 5:-Plot of $(\alpha h v)^{2}$ versus photon energy $(h v)$ for undoped NaAP, LA-NaAP and LT-NaAP Crystals

\section{Photoluminescence spectroscopic studies:-}

Photoluminescence emission spectra of the crystals were recorded using a Perkin Elmer LS-55 spectrometer within the wavelength range of 400 to $700 \mathrm{~nm}$ at room temperature. Undoped NaAP, LA-NaAP and LT-NaAP crystals are excited at a wavelength of $300 \mathrm{~nm}$. The PL spectra of undoped NaAP, LA-NaAP and LT-NaAP crystals are shown in Fig. 6(a-c) are measured in the range $360-570 \mathrm{~nm}$. A good fit of six peaks $(370 \mathrm{~nm}, 409 \mathrm{~nm}, 433 \mathrm{~nm}, 452 \mathrm{~nm}$, $474 \mathrm{~nm}$ and $500 \mathrm{~nm}),(371 \mathrm{~nm}, 399 \mathrm{~nm}, 430 \mathrm{~nm}, 464 \mathrm{~nm}, 482 \mathrm{~nm}$ and $489 \mathrm{~nm})(393 \mathrm{~nm}, 401 \mathrm{~nm}, 428 \mathrm{~nm}, 437 \mathrm{~nm}$, $474 \mathrm{~nm}$ and $491 \mathrm{~nm}$ ) is made using Gaussian function for undoped NaAP, LT-NaAP and LA-NaAP crystals respectively. The six peaks are observed in the UV and visible region. The emission spectra of undoped NaAP crystals peaks at $370 \mathrm{~nm}, 409 \mathrm{~nm}, 433 \mathrm{~nm}, 452 \mathrm{~nm}, 474 \mathrm{~nm}$ and $500 \mathrm{~nm}$ represent the near band edge emission, violet emission, three blue emissions and green emission respectively. LA-NaAP and LT-NaAP crystals show a small blue shift as compared to the undoped NaAP crystal. This may be due to the incorporation of amino acid in the NaAP crystals. Among this blue shift may occur from different origins, such as electron-phonon coupling, lattice distortion, localization of charge carriers owing to interface effects and point defects. In PL spectra the defect level emission was reduced LA-NaAP and LT-NaAP crystals. The optoelectronic properties mainly depend the reduction of defect level in material, which influenced by electron-phonon coupling interaction. In this investigation, LT-NaAP defect level was decreased as compared to undoped NaAP and LA-NaAP. From PL analysis, the strong PL emission of LT-NaAP crystal may find potential applications in optoelectronic devices [18]. 

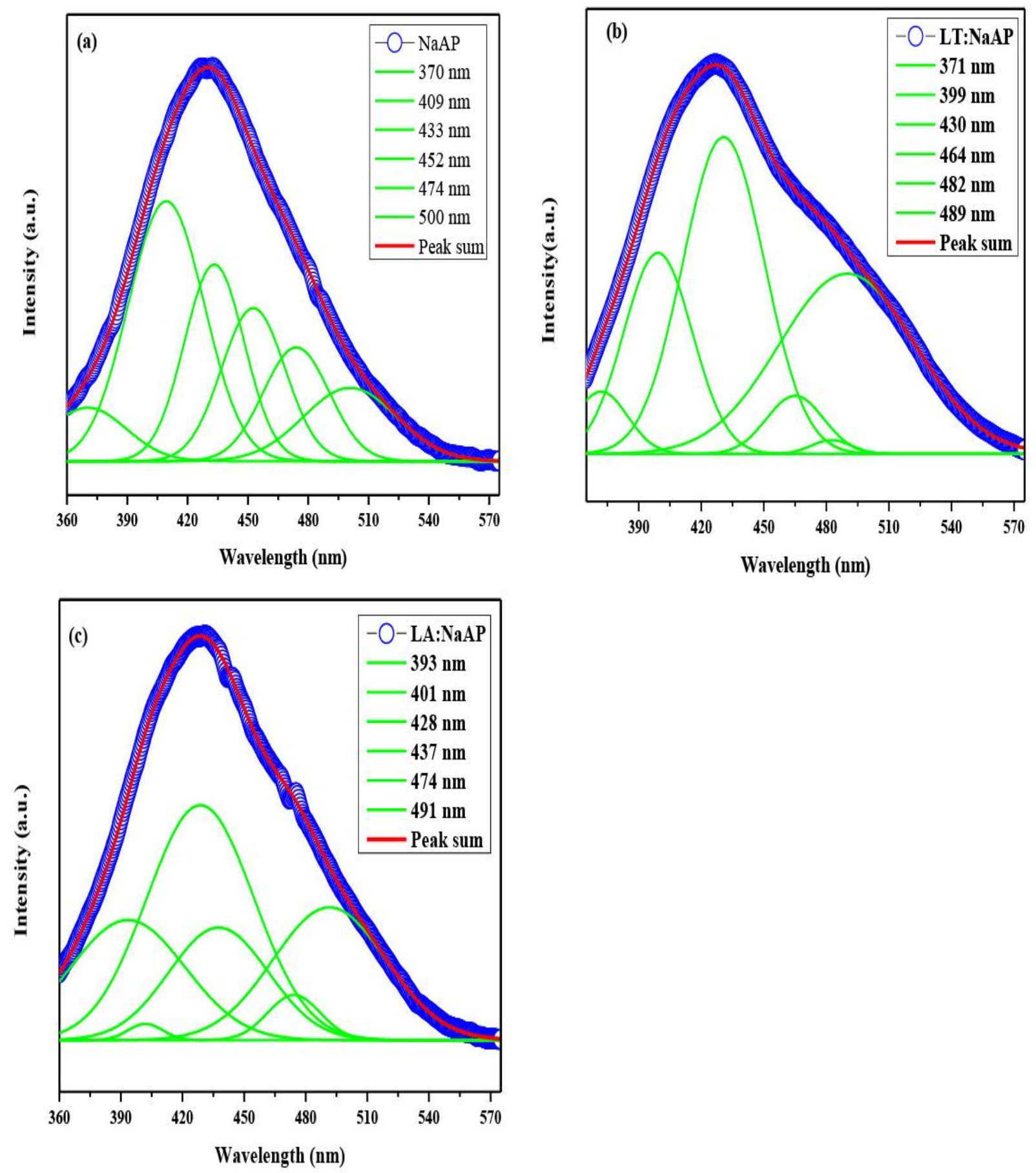

Figure 6(a-c):- PL spectrum of undoped NaAP, LT-NaAP crystal \&LA-NaAP crystals

Vicker's microhardness Analysis:-

The significant property of any device material is its mechanical strength depicted by its hardness and the hardness of a material is a measure of its resistance to plastic deformation [19]. Practically, hardness is the resistance offered by a material to localized plastic deformation caused by scratching or by indentation [20].The permanent deformation can be achieved by indentation, bending, scratching or cutting [21]. The hardness of the crystal contains information about the strength, molecular bindings, yield strength and elastic constants of the material. The Mechanical property of the grown crystals has been studied using Leitz-Wetzler microhardness tester fitted with a Vickers's diamond pyramidal indenter. A well-polished the grown crystals have been placed on the platform of 
Vickers microhardness tester and loads of different magnitudes have been applied in a fixed interval of time. Vicker's microhardness number $\mathrm{H}_{\mathrm{V}}$, was calculated from the following equation (3)

$\mathrm{H}_{\mathrm{V}}=1.8544 \mathrm{P} / \mathrm{d}^{2} \quad\left(\mathrm{Kg} / \mathrm{mm}^{2}\right)$

Where ' $\mathrm{P}$ ' is the applied load $(\mathrm{kg})$, ' $\mathrm{d}$ ' is the diagonal length of indentation impression $(\mathrm{mm})$ and 1.8544 is a constant of the geometrical factor for the diamond pyramid. A plot between $\mathrm{H}_{\mathrm{V}}$ and the load is showed in Fig 7 . 1

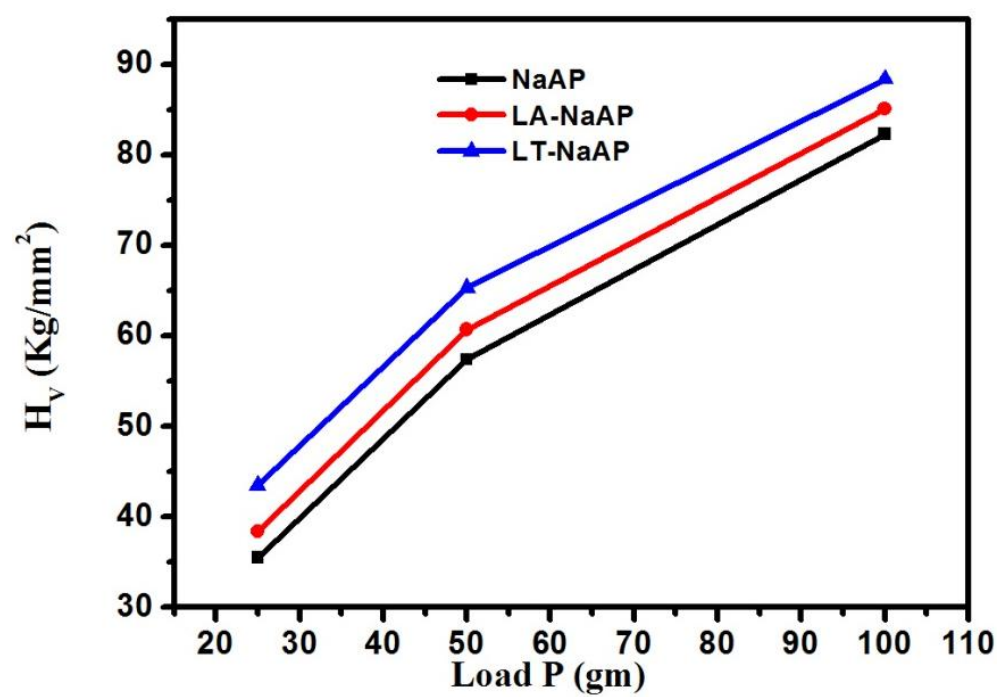

Figure 7:- Load vs Hv plot of undoped NaAP, LA-NaAP \& LT-NaAP crystals

It shows that $\mathrm{Hv}$ increases with increase in load. This can be considered as the reflection of Reverse Indentation Size Effect (RISE) [22-24]. Under this condition, the material undergoes a relaxation that involves a release of the indentation stress along the surface away from the indentation size, which may be because of the crack formation, dislocation activity, and/or elastic deformation of the tip of the indenter [25].

The effect of RISE can be related to energy loss owing to the cracking of the specimen during indentation [26]. Because of cracking, a fraction of energy is spent in crack propagation resulting in small indentation size.

The relation connecting the applied load ' $\mathrm{P}$ ' and diagonal length ' $\mathrm{d}$ ' of the indenter is given by Meyer's law in equation (4)

$\mathrm{P}=\mathrm{k}_{1} \mathrm{~d}^{\mathrm{n}}$

Taking log on both sides of Equation (4), we get

$\log \mathrm{P}=\log \mathrm{k}_{1}+\mathrm{n} \log \mathrm{d}$

Where ' $\mathrm{n}$ ' is Meyer's index (work hardening coefficient) and $\mathrm{k}_{1}$ is a constant of the material. The plot between $\log \mathrm{P}$ and $\log d$ as shown in Figure 8(a-c) is a straight line (after least square fitting) which is in good agreement with Meyer's law. The slope of the graph gives the value of ' $n$ ' and it is found to be 4.4, 3.0 and 2.6 for undoped NaAP, LA-NaAP and LT-NaAP crystals respectively. From the observations made by Onitsch [27] and Hanneman [28], the ' $\mathrm{n}$ ' value lies between 1 and 1.6 for moderately hard materials, and it is more than 1.6 for soft materials. Hence the grown crystals belong to a soft material category. 

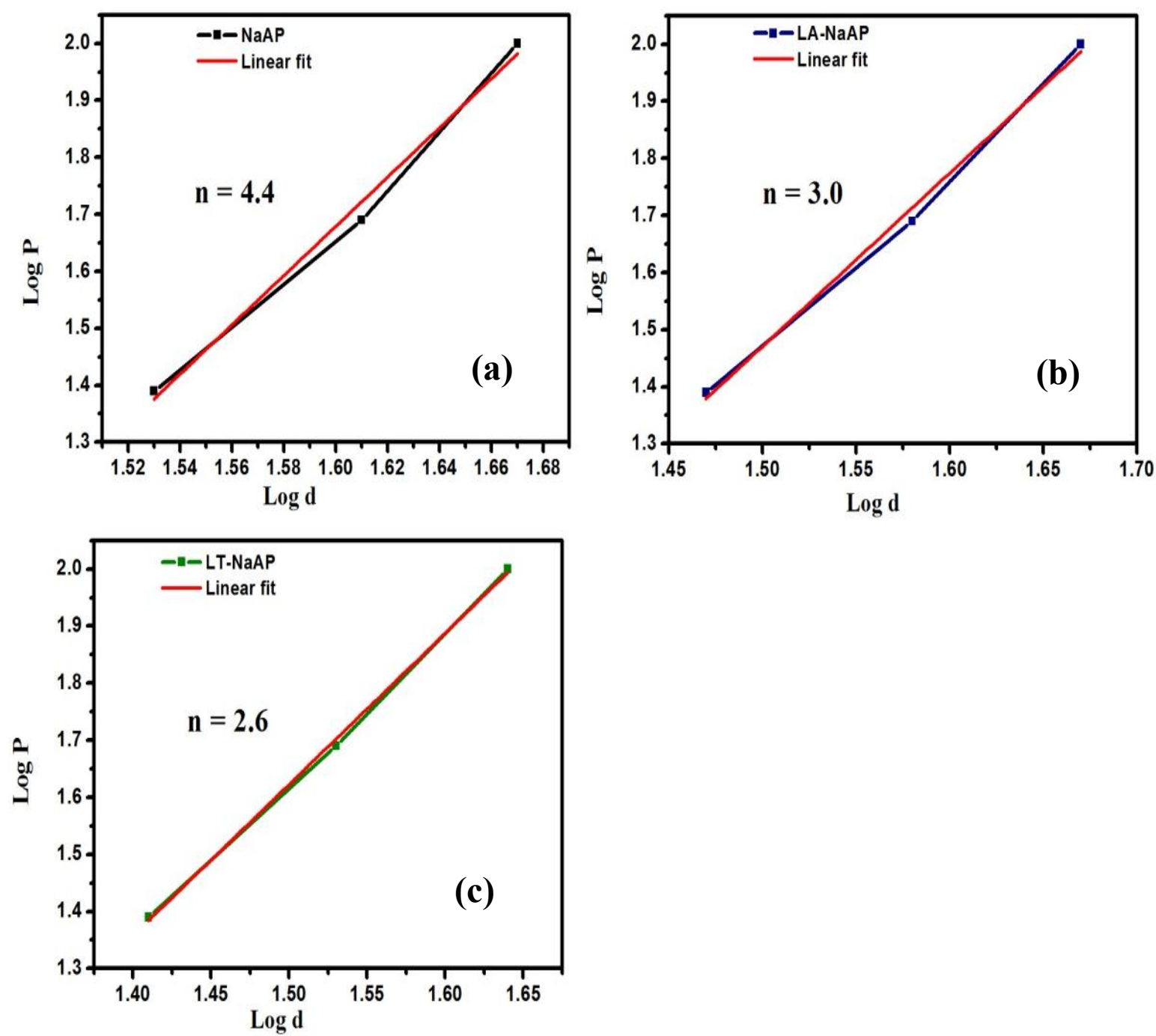

Figure 8 (a-c):- Log P vs Log d plot of undoped, LA-NaAP \& LT-NaAP crystals

Second harmonic generation Analysis:-

The SHG property of undoped, LA-NaAP \& LT-NaAP crystals was tested by the Kurtz and Perry powder method [29] and the fundamental beam of wavelength $1064 \mathrm{~nm}$ by a Q-switched Nd:YAG laser with a pulse energy 3 $\mathrm{mJ} / \mathrm{pulse}$, pulse width $8 \mathrm{~ns}$, and repetition rate $10 \mathrm{~Hz}$ was employed. The phenyl ring and $\pi$-electron system in NaAP crystal are responsible for their NLO efficiency. The measured outputs for undoped NaAP, LA-NaAP and LT-NaAP crystals were $123 \mathrm{mV}, 139 \mathrm{mV}$ and $151 \mathrm{mV}$ respectively. This indicates that the SHG conversion efficiency of LT-NaAP crystal is 1.88 times that of pure KDP crystal (Table 3). The output was confirmed by the emission of green radiation from the sample which indicates that the title material possesses NLO property. Hence LT-NaAP crystal is exploited for the generation of green emission radiation. Apart from this LT-NaAP crystal is best suited in the NLO and optoelectronic device fabrications.

Table 3:- Values of SHG efficiency for undoped and amino acids doped NaAP crystals

\begin{tabular}{|c|c|c|}
\hline Sample Name & SHG efficiency $(\mathbf{m V})$ & Relative efficiency with respective to KDP \\
\hline Undoped NaAP & 123 & 1.53 \\
\hline LA-NaAP & 139 & 1.74 \\
\hline LT-NaAP & 151 & 1.88 \\
\hline
\end{tabular}




\section{Antibacterial Analysis:-}

The antibacterial activity of the undoped NaAP (S1), LA-NaAP (S2) and LT-NaAP (S3) was carried out by the well diffusion method against the bacterial strains of $S$. pneumonia, E. coli and K. pneumonia on Mueller hinton agar, according to the Clinical and Laboratory Standards Institute (CLSI) [30]. The media plate's Mueller hinton agar (MHA) was streaked with bacteria 2-3 times by rotating the plate at $60^{\circ}$ angles for each streak to ensure the homogeneous distribution of the inoculums. After inoculation, discs (6 mm Hi-Media) loaded $10 \mu \mathrm{g} / \mathrm{ml} \mathrm{of} \mathrm{the} \mathrm{test}$ samples were placed on the bacteria-seeded well plates using micropipettes and the plates were then incubated at $37^{\circ} \mathrm{C}$ for $24 \mathrm{~h}$. The inhibition zone around the well was measured and recorded. Erythromycin (Hi-Media) was used as the positive controls against $S$. pneumonia, E. coli and $K$. pneumonia bacteria respectively. The positive controls results were as compared to that of the efficacy of the test NaAP (S1), LA-NaAP (S2) and LT-NaAP (S3) samples. The antibacterial activity results of undoped NaAP, LA-NaAP and LT-NaAP samples are shown in Fig. 9. Fig 10 shows the size of the zone of inhibition and antibacterial activity formed around each well concentration $(3 \mathrm{mg} / \mathrm{ml})$ of undoped NaAP, LA- NaAP and LT-NaAP loaded with test samples. Antibacterial results showed that all the grown samples possess biological activity [31,32]. The antibacterial activity of undoped NaAP, LA-NaAP and LT-NaAP samples zone inhibition values are increased as compared to that of commercial antibiotic erythromycin.
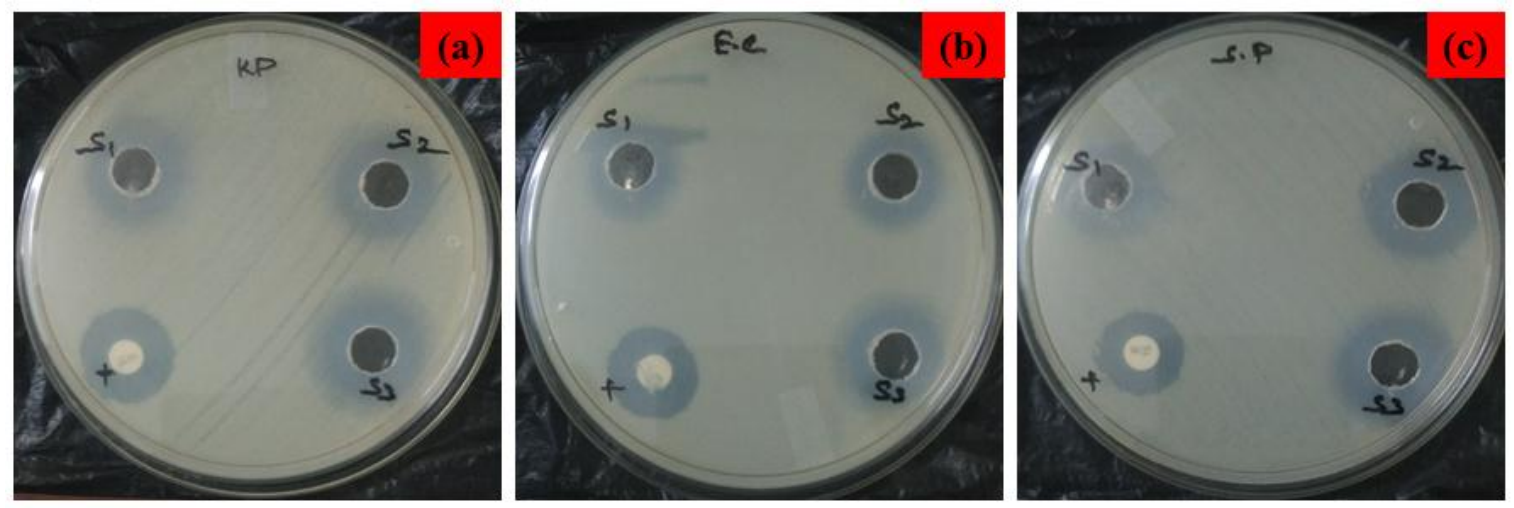

Figure 9:- The progressive antibacterial activity of undoped NaAP (S1), LA-NaAP (S2) and LT-NaAP (S3) against towards K. pneumonia, E. coli and S. pneumonia

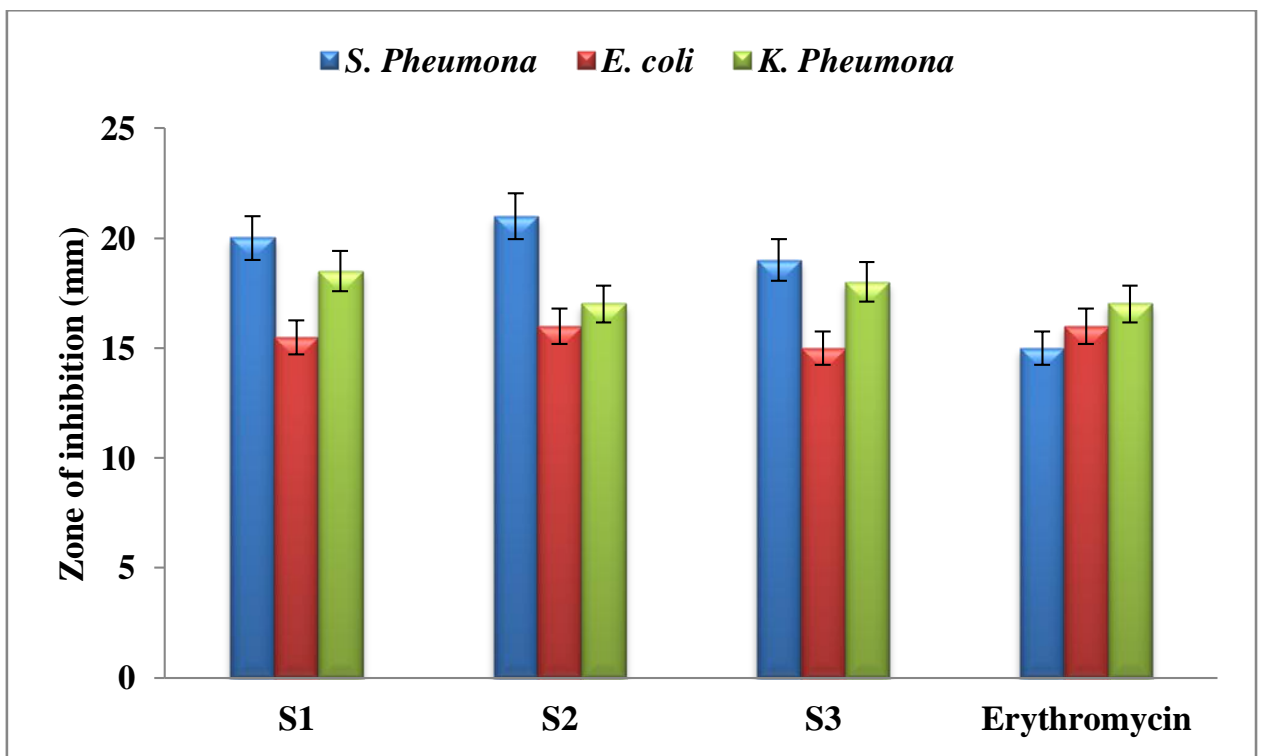

Figure 10:- The size of the zone of inhibition formed around each well, loaded with test samples, indicating the antibacterial activity towards $S$. pneumonia, E. coli and $K$. pneumonia for undoped NaAP (S1), LA-NaAP (S2) and LT-NaAP (S3) samples 


\section{Conclusion:-}

Undoped NaAP, LA-NaAP and LT-NaAP crystals were grown successfully by slow evaporation technique. Single $\mathrm{X}$-ray diffraction revealed that all the grown crystals possess orthorhombic crystal structure and revealed lattice distortion due to impurities. The crystallinity nature of the grown crystals was analyzed by powder X-ray diffraction analysis. Various functional groups of the grown crystals were identified by FT-IR spectral analysis. The optical transparency, optical band gap, relative second harmonic generation efficiency and mechanical properties are found to be enhanced due to L-threonine dopant. From the PL spectra, the defect level of LT-NaAP crystal was decreased as compared to that of undoped NaAP and LA-NaAP crystals. These results provided strong support for the further development of extensive optoelectronic device applications. The zone inhibition values of undoped NaAP, LA-NaAP and LT-NaAP samples are increased as compared to that of commercial antibiotic erythromycin by antibacterial analysis. By assessing the present results it may suggest that L-threonine doped NaAP crystal is a potential candidate for optoelectronic device applications.

\section{Acknowledgment:-}

The authors are grateful to Dr. Babu Varghese, Head, SAIF IIT-Madras, B.S. Abdur Rahman University, Chennai and St. Joseph's College, Trichy for providing characterization facilities and antibacterial studies were done by KIRND Institute of Research and Development PVT LTD, Tiruchirappalli.

\section{References:-}

1. R.A. Laudise (1970). The growth of single crystals, Prentice Hall, Eagle wood Cliffs, New Jersy.

2. R.A. Laudise (1975). in: R. Ueda, J.B. Mullin (Eds.), Crystal growth and characterization, North-Holland publishing Co.

3. D.S. Chemla, J. Zyss (1987). Non-linear Optical Properties of organic molecules and crystals, Vol 1-2, Academic Press, Orlando.

4. S.P. Velsko (1990). Laser Program Annual Report, Lawrence UCRL-JC 105000, Lawrence Livermore National Laboratory, Livermore, California.

5. L.F.Warren (1990). Society for the Advancement of Material and Process Engineeing, Covina, Ca., 1990 in: R.E. Allred, R.J. Martinez, W.D.Wischmann (Eds.), Proceedings of the Fourth International SAMPE Electronics Conference, $\quad$ 4, 388-396.

6. G. Xing, M. Jiang, Z. Sao, D. Xu (1987). Bis(thiourea) cadmium chloride (BTCC) a novel nonlinear optical crystal of organometallic complex, Chin. J. Lasers 14, 302-308.

7. Christer B. Aakeröy, Peter B. Hitchcock, Brian D. Moyle, Kenneth R. Seddon (1989). A novel class of salts for second harmonic generation,J. Chem. Soc. Chem. Commun, 1856-1859.

8. R. Srineevasan, R. Rajasekaran (2013). Growth and optical studies of 2-aminopyridine bis thiourea zinc sulphate (2-APTZS) single crystals for NLO applications, J. Mol. Struct, 1048, 238-243.

9. R. Rajasekaran, P.M. Usharee, R. Jayavel, P. Ramasamy (2001). Growth and characterization of zinc thiourea chloride (ZTC): a semiorganic nonlinear optical crystal, J. Cryst. Growth 229, $563-567$.

10. R. Bairava Ganesh, V. Kannan, K. Meera, N.P. Rajesh, P. Ramasamy (2005). Synthesis, growth and characterization of a new nonlinear optical crystal sodium acid phthalate, J. Crystal growth. 282, 429-433. S. Krishnan, C. Justin Raj, S. Dinakaran, R. Uthrakumar, R. Robert, S. Jerome Das (2008). Optical, thermal, dielectric and ferroelectric behaviour of sodium acid phthalate (SAP) single crystals, J. Phys. Chem. Solids, 69, 2883-2887.

11. V. Krishnakumar, R. Nagalakshmi (2005). Crystal growth and vibrational spectroscopic studies of the semiorganic non-linear optical crystal-bisthiourea zinc chloride, Spectrochim Acta A, 61, 499-507.

12. V. Krishnakumar, R. John Xavier (2005). FT Raman and FT-IR spectral studies of 3-mercapto-1,2,4-triazole, Spectrochim Acta A, 60, 709-714.

13. Roshan, S. Cyriac Joseph, M.A Ittyachen (2001). Growth and characterization of a new metal-organic crystal: potassium thiourea bromide, Mater. Lett. 49, 299-302.

14. V.V. Maheswaran, S. Sherwood, J.N. Bhat (1997). Crystal growth and physical characterization of the semiorganic bis(thiourea) cadmium chloride, J. Crystal growth, 179, 605-610.

15. N.Tigau, V. Ciupina, G. Prodana, GI. Rusub, C. Gheorghies, E. Vasile (1967). Influence of thermal annealing in air on the structural and optical properties of amorphous antimony trisulfide thin films, J Optoelectron Adv M, 6, 211-217.

16. E.D.D.O. Ekpunobi and A.J. Okeke (2006). Acad. Open Internet J. 17. 
17. J.X. Wang, S.S. Xie, H.J. Yuan, X.Q. Yan, D.F. Liu, Y. Gao, Z.P. Zhou, L. Song, L.F.Liu, X.W. Zhao, X.Y. Dou, W.Y. Zhou, G. Wang (2004). Synthesis, structure, and photoluminescence of $\mathrm{Zn}_{2} \mathrm{SnO}_{4}$ single-crystal nanobelts and nanorings, Solid State Commun. 131, 435-440. Mott BW (1956). Micro indentation Hardness Testing,Butterworth, London.

18. Subhadra KG,Kishan Rao K, Sirdeshmukh DB (2000). Systematic hardness studies on lithium niobate crystals, Bull Mater Sci, 23,147-150.

19. M. Senthil Pandian, N. Balamurugan, V. Ganesh, PV. Raja Shekar, K. Kishan Rao, P.Ramasamy (2008). Growth of TGS single crystal by conventional and SR method and its analysis on the basis of mechanical, thermal, optical and etching studies, Mater. Lett. 62, 3830-3832.

20. K. Sangwal (2000). On the reverse indentation size effect and microhardness measurement of solids, Mater Chem Phys. 63, 145-152.

21. J. Gong, H. Miao, Z. Zhao, Z. Guan, Zhenduo Guan (2001). Load-dependence of the measured hardness of Ti(C,N)-based cermets, Mater Sci Eng. A, 303, 179-186.

22. B. Basu, NK. Mukhopadhyay, Manisha (2009). Understanding the mechanical properties of hot pressed Badoped S-phase SiAlON ceramics, J Eur Ceram. Soc. 29, 801-811.

23. HS. Güder, E. Sahin, O. Sahina, H. Göçmez, C. Duran, H. Ali Çetinkara (2011) Vickers and Knoop Indentation Microhardness Study of $\beta$-SiAION Ceramic, Acta. Phys. Pol. A, 120,1026-1033.

24. P. Feltham, R. Banerjee (1992). Theory and application of micro indentation in studies of glide and cracking in single crystals of elemental and compound semiconductors, J Mater Sci, 27, 1626-1632.

25. Onitsch EM (1947). Micro-Hardness testing, Mikroscopie 2, 131-151.

26. Hanneman M (1941). Indentation size effect and microhardness study, Metall Manch, 23, 135-139.

27. S.K. Kurtz and T.T. Perry (1968). A Powder Technique for the Evaluation of Nonlinear Optical Materials, J. Appld. Phys, 39, 3798-3812.

28. Gerard D. Wright (2000). Resisting resistance: new chemical strategies for battling superbugs, Chem. Biol, 7, R127-R137.

29. V. Renuga (2014). Synthesis, Characterization and Biological Activity of Pure and Metal Ions Doped L-Proline Amino Acid. Int J Sci Res Pub, 4, 1-9.

30. T. Prasanyaa, M. Haris, V. Jayaramakrishnan, M. Amgalan, V. Mathivanan (2013). Synthesis, characterization and anti-microbial activity of pure, $\mathrm{Cu}^{2+}$ and $\mathrm{Cd}^{2+}$ doped organic NLO L-arginine trifluoroacetate single crystals. Phys. Scr, 88, 045403-045408. 